\title{
(2) OPEN ACCESS \\ A rationale and framework for seeking remote electronic or phone consent approval in endovascular stroke trials - special relevance in the COVID-19 environment and beyond
}

\author{
Ansaar T Rai [i] , ${ }^{1}$ Donald Frei $\left[{ }^{2}\right.$
}

${ }^{1}$ Neuroradiology, Rockefeller Neurosciences Institute, West Virginia University Robert C Byrd Health Sciences Center, Morgantown, West Virginia, USA

${ }^{2}$ Interventional Neuroradiology, Radiology Imaging Associates, Englewood, Colorado, USA

\section{Correspondence to}

Dr Ansaar T Rai, Neuroradiology, Rockefeller Neurosciences

Institute, West Virginia

University Robert C Byrd Health

Sciences Center, Morgantown, WV 26505-4024, USA; ansaar. rai@gmail.com

Received 22 April 2020 Revised 27 April 2020 Accepted 28 April 2020

Published Online First 7 May 2020
Check for updates

(C) Author(s) (or their employer(s)) 2020. Re-use permitted under CC BY-NC. No commercial re-use. See rights and permissions. Published by BMJ.

To cite: Rai AT, Frei D. J Neurolntervent Surg 2020;12:654-657.

\section{ABSTRACT \\ Background Enrollment in time-sensitive endovascular} stroke trials can be challenging because of an inability to consent a debilitated patient. Often the legally authorized representative is not on site. Remote consent procedures in the US are inconsistent with the majority of sites shunning these approaches. The current pandemic with visitor restrictions highlights the need for enhancing these options.

Methods Remote electronic and phone consent procedures specifically for endovascular stroke trials from two comprehensive stroke centers (CSC) are presented. An overview of the genesis of informed consent procedures in the US is also included.

Results The two CSCs identified as Institution- 1 and Institution-2 are large tertiary systems. Institution- 1 is a non-profit university-affiliated academic medical center in rural geography. Institution-2 is an HCA hospital in an urban environment. Both serve patients through a spoke-and-hub network, have participated in multiple randomized endovascular stroke trials, and have successfully used these remote options for enrollment. A tiered approach is employed at both institutions with an emphasis on obtaining informed consent in person and resorting to alternatives methods when efforts to that are unsuccessful. A rationale for electronic and phone consent is included, followed by step-by-step illustration of the process at each institution.

Conclusion Two examples of remote electronic or phone consent procedures from institutions in different geographic environments and organization structures demonstrate that these options can be successfully used for enrollment in stroke trials. The current pandemic highlights the need to enhance these approaches while maintaining appropriate adherence to ethical and legal frameworks.

\section{INTRODUCTION}

The National Commission for the Protection of Human Subjects of Biomedical and Behavioral Research was created in 1974. The Commission's charge was to develop guidelines for human subject research based on ethical principles that would underpin such research. The output of the Commission after in-depth discussions over the next 4 years was the Belmont Report, which was published in the Federal Register in April 1979. ${ }^{1}$ Three core ethical principles identified by the Belmont Report were respect for persons, beneficence, and justice. The guidance for obtaining legally effective informed consent provided by the US Department of Health and Human Services code of federal regulations (CFR) Title 45 (Public Welfare), Part 46 (Protection of Human Subjects) - "45 CFR part 46" is grounded in the Belmont Report's first principle of "respect for persons". (https://www.ecfr.gov/cgibin/ retrieveECFR?gp $=\& S I D=83 \mathrm{~cd} 09 \mathrm{e} 1 \mathrm{c} 0 \mathrm{f} 5 \mathrm{c} 6937 \mathrm{~cd} 9$ d7513160fc3f\&pitd $=20180719 \& n=p t 45.1 .46 \&$ $\mathrm{r}=$ PART \& $\mathrm{ty}=\mathrm{HTML}$ ).

Since then, obtaining appropriate informed consent from the patient or next of kin is a key ethical and medicolegal requirement - and in the US it is the right of any patient prior to undergoing an invasive procedure. ${ }^{2}$ The informed consent obtained from the subject or the subject's legally authorized representative (LAR) is a process that needs to be followed not only in letter but in spirit. The food and drug administration (FDA) final rule regarding exception to informed consent in emergency medical research was published in $1996^{3}$ and contains explicit criteria governing conditions in which critically ill patients can have access to potentially lifesaving (but unproven) therapies. This has been recently updated to include new guidance on obtaining electronic informed consent. ${ }^{4}$ Approval of remote or electronic informed consent protocols or exception for informed consent is determined by the local institutional review boards (IRB) and is dependent on their comfort and level of understanding vis a vis informed consent procedures especially in an emergency setting. ${ }^{5}$

Acute ischemic stroke secondary to large vessel occlusion constitutes a medical emergency requiring prompt intervention to reperfuse the brain. These interventions are now evidence-based and constitute standard of care. Further progress is dependent on continuous research and clinical trials. Large vessel stroke patients represent a unique group debilitated by their neurological deficits and often unable to comprehend their state or surroundings. Transferred by emergency medical services and often from nursing homes or independent senior living facilities these patients are not in immediate touch with their families. Obtaining a face-to-face informed consent from a next of kin for enrollment in a clinical trial highly dependent on time to treatment is often not possible and thus these patients 
are excluded from enrollment. This may lead to prolongation of the trial and also introduces a certain bias by enrolling only those patients whose relatives are readily available and potentially have a stronger family structure which may impact outcomes. ${ }^{6}$

The current COVID-19 pandemic with restricted limited visitor policy in most institutions is particularly an environment that may benefit from evolution in consent procedures for now and for the future. This brief article describes the remote consent procedure adopted at two institutions involved in endovascular trials for acute ischemic stroke. The article also presents a review of literature and remote consenting methods adopted in other clinical trials.

\section{METHODS}

We describe remote consent processes for endovascular stroke trials at two institutions. These are large comprehensive stroke centers that have participated in multiple acute ischemic stroke trials. The process at both these institutions was developed to allow for enrollment of eligible patients without causing any delay in treatment.

\section{RESULTS}

\section{Institution-1}

The first institution is a university affiliated tertiary referral comprehensive stroke center covering a large rural geography with a relatively low population density. Often a stroke patient arrives or is transferred much ahead of the family and next of kin, limiting the capacity to do in-person informed consent. The remote consent process was initially developed several years ago to improve enrollment in the stroke trials and since then has been a part of the protocol for all endovascular stroke trials. The same pathway will be adopted in the current COVID environment and beyond the acute pandemic when families may not be allowed to accompany a patient. The rationale for the proposal to the IRB was based on the following key points:

- Endovascular stroke therapy is a time-sensitive procedure with proven efficacy.

- As standard of care, emergent endovascular therapy for large vessels strokes is routinely performed without obtaining informed consent if a next of kin is unavailable. This is similar to other acute lifesaving interventions.

- The time to recanalization is benefitted by having prescribed streamline processes aimed at efficiency.

- A patient suffering from a large vessel stroke is often unable to consent for him or herself.

- The families of many patients transferred for emergent endovascular stroke therapy do not accompany the patients during transfer and are often only available by phone.

- The option to not enroll the patient is always there and at times may be the only option to follow. However, transformational changes in any disease management hinge on the ability to enroll patients in FDA-approved clinical trials with appropriate adherence to the code of federal regulations.

The following tiered methodology was approved by the IRB and successfully used to enroll patients in whom face-to-face consent from the next of kin could not be obtained.

- An IRB-approved one-page consent script summarizing the trial was used (sample in figure 1). The rationale of the onepage script was to save time. The preferred procedure would be to make every reasonable effort in obtaining face-to-face consent from the next of kin if the patient cannot consent. The enrollment may be initiated after the LAR signs the

\section{Consent Form Script:}

Ask the MPOA: Would you like to receive this document by email, as a cell phone image document, by fax or would you like me to discuss it with you over the phone?

The treating physicians have determined that you (or the patient for whom you are the legal guardian) have had a stroke caused by a blood clot blocking the flow of blood to an area in your brain. This is called an Acute Ischemic Stroke.

We want to know if you would you be interested in participating in a study comparing the first attempt (approach) to removing the blood clot by

for treating your stroke. or the use of a medical device called

The devices in both groups are FDA approved. Both approaches are considered standard of care and do not involve investigational devices. Because we do not know which of the 2 approaches treatments.

If you agree to participate in this research you will be randomized to one of two treatment groups selected by chance, as if by tossing a coin. Whether or not you participate, you will receive the standard of care that all stroke patients receive.

Please note the side effects listed on pages 3 of 6 of the consent forms, and remember that unknown side effects not listed could occur. The biggest risk is bleeding in the brain.

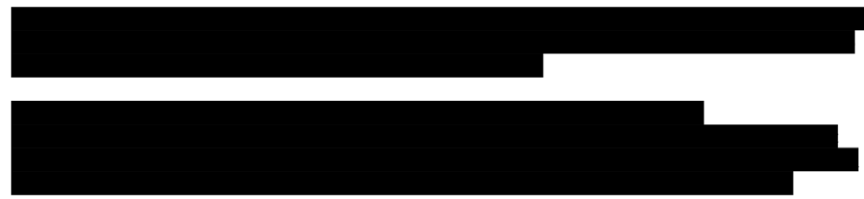

Do you have any questions?

$\checkmark$ Check if Phone Consent. Record date, name \& relationship of person you spoke to:

Signature Date

Investigator or Co-Investigator Signature Date

Witness Signature Date

Figure 1 A sample of a one-page consent form script is presented: study related information has been redacted.

one-page consent. The LAR or next of kin can then sign the full IRB-approved consent form.

- If the patient cannot consent and the LAR is not on site at the arrival of the patient, one of the following options will be followed depending on the LAR's location:

a. LAR is at the outlying transferring hospital: The onepage consent form script will be faxed and reviewed with the LAR over the phone. Similar to above, the complete consent form will be faxed for the representative to sign and fax back to be countersigned by the investigator.

b. LAR is at home or en-route with a driver: If the LAR is at home or en route with a driver, an electronic version or pre-prepared pdf picture of the one-page consent form script will be sent and reviewed with the patient. If the LAR verbally agrees to the trial, enrollment will be initiated. Complete signed consent will be obtained as soon as the LAR is on site.

c. If the LAR is driving: The LAR will be asked to pull to the side of the road or seek a parking space for the phone conversation. The procedure as described above will be followed. The enrollment will be initiated if the LAR verbally agrees and complete consent will be obtained once the LAR is on site.

- For either option (b) or (c) if the LAR does not have the ability to receive an electronic version or picture on the phone, verbal consent will be obtained after reviewing and explaining the one-page consent script.

- For both the scenarios in options (b) and (c) if the LAR agrees over the phone but changes his/her mind on arrival and does not want trial participation to continue, the enrollment will be annulled and the sponsor will be notified. 
- The remote consent process applies to emergent endovascular stroke trials as long as the standard of thrombectomy care is not at risk of being excluded. This could be trials that randomize between two thrombectomy options (eg, aspiration vs stent-retrievers) or trials that randomize between medical management and endovascular therapy for large vessels strokes where endovascular therapy is not proven and may be otherwise not offered, such as patients with poor imaging profile.

\section{Institution-2}

Similar to the first one, the second institution is also a large comprehensive stroke center but contrary to the first one it is an HCA hospital in an urban environment. The institution is the prime referral base for a large geography and population. The institution developed a remote consent protocol for an ongoing endovascular stroke trial specifically to allow enrollment during the COVID-19 pandemic. The rationale was similar to that described for the previous institution. The tiered remote consent process in case face-to-face consent cannot be obtained is described below:

- Physician introduces the study to the LAR over the phone.

- If LAR is amenable, physician confirms the best number at which the research coordinator can reach them.

- Coordinator calls the LAR and asks how they would like to receive an electronic copy of the ICF, by email or text.

\section{If the LAR has the ability to print}

i. Coordinator asks them to print the consent prior to reviewing it with them and calls back when it has been printed, as needed.

ii. Once LAR has a printed copy, the coordinator goes through the consent and the HIPAA authorization forms, reminding the LAR to initial on each page, then sign on the appropriate line, date and timestamp both documents.

iii. LAR scans (if able) or takes a picture of the signature page and sends to coordinator (email is preferred, text message is acceptable), to be saved as a PDF. The original version will be mailed back to the site.

iv. Coordinator signs, dates, and timestamps the copy that they have and verifies the copy sent to them. The coordinator completes the ICF process form.

v. Coordinator makes a complete copy (with two signature pages, one with the patient's and one with the coordinator's) by printing patient signature page and adding to their printed copy of the consent and HIPAA authorization forms. Coordinator then scans this as the complete PDF. Coordinator sends to the LAR by requested method (email or mail).

vi. A single copy of the consent and HIPAA authorization forms is also sent to the hospital medical records department via email for inclusion in the patient chart.

\section{If the LAR is unable to print}

i. Coordinator reviews the consent and HIPAA authorization forms with the LAR and documents their verbal consent both on the consent process checklist and by signing, dating, and timestamping the copy that they have in order to meet protocol-required timing of informed consent.

ii. A copy of the consent and HIPAA authorization forms is then emailed or mailed to the LAR.

iii. Once received, the coordinator reviews the consent and HIPAA authorization forms and verifies that the LAR has no additional questions, reviews where the LAR needs to initial and sign.

iv. LAR scans (if able) or takes a picture of the signature page and sends to coordinator (email is preferred, text message is acceptable), to be saved as a PDF. The original version will be mailed back to site.

v. Coordinator makes a note on the ICF that consent was rereviewed with the LAR, then initials and dates.

vi. Coordinator makes a complete copy (with two signature pages, one with the patient's and one with the coordinator's) by printing patient signature page and adding to their printed copy of the consent and HIPAA authorization forms. Coordinator then scans this as the complete PDF. Coordinator sends to the LAR by requested method (email or mail).

vii. A single copy of the consent and HIPAA authorization forms is also sent to the hospital medical records department via email for inclusion in the patient chart.

viii. No medical records will be accessed, and no data will be entered into the study Electronic Data Capture (EDC) until a signed consent is in place.

\section{DISCUSSION}

The article describes remote consent processes adopted for use in two centers for emergent endovascular stroke trials. The critical requirement for obtaining remote consent is to balance adherence to core ethical principles with the capacity to enroll neurologically debilitated patients who may not be able to consent themselves. Endovascular stroke therapy is a standard of care for large vessels strokes, future trials may assess different treatment options for recanalization or may access subgroups for whom this therapy is not of proven efficacy. These groups can include large vessel stroke patients with a higher core infarct on imaging, second order cerebrovascular occlusions, or large vessels strokes with relatively minor neurological deficits.

Physician phone elicitation of consent in the field has been shown to be feasible and effective in increasing enrollment, especially for time-sensitive trials such as acute ischemic stroke. ${ }^{7}$ The EXTEND-IA ${ }^{8}$ allowed the option of verbal phone consent from a LAR if in-person consent could not be obtained and the ESCAPE $^{9}$ trial allowed for waiver or deferral of consent when necessary and where possible according to local IRB approval. The use of a smartphone platform for obtaining electronic informed consent was demonstrated by Haussen et al for enrollment in the DAWN ${ }^{10}$ trial with a follow-up study showing overall acceptable response of the electronic process by the consenters. ${ }^{11}$ The FAST-MAG trial implemented a novel voice over the internet phone system and simultaneous activation of multiple physicians to connect the first available investigator with on-scene first responders and patient or their legally authorized representative. ${ }^{12}$ By facilitating and expediting the informed consent procedure using voice-over-internet phone, the trial was able to enroll a substantial number of patients in the first hour after stroke symptom onset. ${ }^{12}{ }^{13}$ Patient comprehension of informed consent has been shown to be non-inferior to face-to-face consent in emergency medicine trials. ${ }^{14}$

Technological progress over the past decade has transformed communications. Widespread availability of smart phones, tablets, faster networks, and targeted applications are allowing physicians to remotely communicate with their patients and even conduct a virtual clinic visit. The current COVID-19 pandemic has highlighted these modes of communication with an emphasis on telemedicine to cope with the lack of in-person interactions. ${ }^{15} 16$ Further enhancement of these approaches may require an update 
of the health informatics infrastructure ${ }^{17}$ and a renewed evaluation of these possibilities by IRBs.

\section{CONCLUSION}

This article illustrates two examples for remote electronic or phone consent procedures adopted by comprehensive stroke centers to sustain enrollment in emergent endovascular stroke trials. This is particularly pertinent during the COVID-19 pandemic where many hospitals do not allow visitors or severely limit the ability of LAR or LAR admittance into the hospital. There are other examples in the literature on how these processes can be developed, keeping in mind the federal guidance for informed consent, the ethical principles at stake, and the unique settings of a clinical trial. The onus of balancing these criteria is on the sponsor, the principal investigators, and on the local IRB. The current pandemic has presented an opportunity to evaluate and enhance electronic communication tools and update the rules of conducting ethical clinical research in compliance with the code of federal regulations.

\section{Twitter AnsaarT Rai @Ansaar_Rai and Donald Frei @donfreimd}

Contributors Both authors contributed to the manuscript.

Funding The authors have not declared a specific grant for this research from any funding agency in the public, commercial, or not-for-profit sectors.

Competing interests None declared.

Patient consent for publication Not required.

Ethics approval An ethics approval was not required because the study does not involve any human subjects, data collection, or protected health information.

Provenance and peer review Not commissioned; externally peer reviewed.

Data availability statement Data sharing not applicable as no datasets generated and/or analyzed for this study. There are no datasets for the study.

Open access This is an open access article distributed in accordance with the Creative Commons Attribution Non Commercial (CC BY-NC 4.0) license, which permits others to distribute, remix, adapt, build upon this work non-commercially, and license their derivative works on different terms, provided the original work is properly cited, appropriate credit is given, any changes made indicated, and the use is non-commercial. See: http://creativecommons.org/licenses/by-nc/4.0/.

ORCID iDs

Ansaar T Rai http://orcid.org/0000-0001-9864-4805

Donald Frei http://orcid.org/0000-0002-7811-5030

\section{REFERENCES}

1 Protection of human subjects; Belmont report: notice of report for public comment. Fed Regist 1979:44:23191-7.

2 Perrenoud B, Velonaki V-S, Bodenmann P, et al. The effectiveness of health literacy interventions on the informed consent process of health care users: a systematic review protocol. JBI Database System Rev Implement Rep 2015;13:82-94.

3 Protection of human subjects; informed consent - FDA. Final rule. Fed Regist 1996;61:51498-533.

4 New guidance on electronic informed consent. Cancer Discov 2017:7:0F4.

5 Ernst AA, Fish S. Exception from informed consent: viewpoint of institutional review boards - balancing risks to subjects, community consultation, and future directions. Acad Emerg Med 2005;12:1050-5.

6 Aron AW, Staff I, Fortunato G, et al. Prestroke living situation and depression contribute to initial stroke severity and stroke recovery. I Stroke Cerebrovasc Dis 2015:24:492-9.

7 Saver JL, Kidwell C, Eckstein M, et al. Physician-investigator phone elicitation of consent in the field: a novel method to obtain explicit informed consent for prehospital clinical research. Prehosp Emerg Care 2006;10:182-5.

8 Campbell BCV, Mitchell PJ, Kleinig TJ, et al. Endovascular therapy for ischemic stroke with perfusion-imaging selection. N Eng/ J Med 2015;372:1009-18.

9 Goyal M, Demchuk AM, Menon BK, et al. Randomized assessment of rapid endovascular treatment of ischemic stroke. N Eng/ J Med 2015;372:1019-30.

10 Haussen DC, Doppelheuer S, Schindler K, et al. Utilization of a smartphone platform for electronic informed consent in acute stroke trials. Stroke 2017:48:3156-60.

11 Haussen DC, Craft L, Doppelheuer S, et al. Legal authorized representative experience with smartphone-based electronic informed consent in an acute stroke trial. J Neurointerv Surg 2020;12:483-5.

12 Sanossian N, Starkman S, Liebeskind DS, et al. Simultaneous ring voice-over-Internet phone system enables rapid physician elicitation of explicit informed consent in prehospital stroke treatment trials. Cerebrovasc Dis 2009;28:539-44.

13 Saver JL, Starkman S, Eckstein M, et al. Methodology of the Field Administration of Stroke Therapy - Magnesium (FAST-MAG) phase 3 trial: Part 2 - prehospital study methods. Int I Stroke 2014;9:220-5.

14 Bobb MR, Van Heukelom PG, Faine BA, et al. Telemedicine provides noninferior research informed consent for remote study enrollment: a randomized controlled trial. Acad Emerg Med 2016;23:759-65.

15 Ohannessian R, Duong TA, Odone A. Global telemedicine implementation and integration within health systems to fight the COVID-19 pandemic: a call to action. JMIR Public Health Surveill 2020;6:e18810.

16 Hollander JE, Carr BG. Virtually perfect? Telemedicine for Covid-19. N Eng/ J Med 2020;382:1679-81.

17 Reeves JJ, Hollandsworth HM, Torriani FJ, et al. Rapid response to COVID-19: health informatics support for outbreak management in an academic health system. J Am Med Inform Assoc 2020. doi:10.1093/jamia/ocaa037. [Epub ahead of print: $24 \mathrm{Mar}$ 2020]. 